\title{
El bandolerismo mexicano como respuesta al contexto socioeconómico porfirista (1877-1893)
}

\section{Mexican banditry in response to the porfirist socioeconomic context (1877-1893)}

\author{
Dr. Pablo Escalante Piña* \\ UNIVERSIDAD PARA EL BIENESTAR BENITO JUÁREZ GARCÍA-ARMERÍA \\ https://doi.org/10.22370/pe.2019.7.2617
}

\section{RESUMEN}

En la presente investigación se aborda el bandolerismo mexicano en un periodo en el cual las circunstancias del contexto histórico fueron coyunturales para la presencia de dicha práctica social. Se plantea como idea central que efectivamente el bandolerismo mexicano fue una de las tantas respuestas al contexto socioeconómico entre 18771893. Para ello, en un primer momento se elabora un balance historiográfico a partir del inicio de la guerra de independencia hasta la década de 1870 . Asimismo, se efectúa la caracterización de la región conocida como el Bajío michoacano, partiendo del supuesto de que el contexto socioeconómico pudo influir en los índices de presencia y permanencia del bandidaje. Finalmente, se realiza la identificación de las gavillas y bandidos que tuvieron lugar en el Estado de Michoacán entre 1877-1893, ello, para delimitar la morfología del bandidaje y la relación que guardaba con el contexto que se estaba viviendo en la sociedad mexicana.

Palabras clave: Bandolerismo, Bandidos, gavillas, porfiriato, socioeconómico

\begin{abstract}
This research addresses Mexican banditry in a period in which the circumstances of the historical context were conjunctural for the presence of said social practice. It is proposed as a central idea that indeed Mexican banditry was one of the many responses to the socioeconomic context between 1877-1893. To do this, at first a historiographical balance is drawn up from the beginning of the war of independence until the 1870s. Likewise, the characterization of the region known as the Michoacán Bajío is carried out, starting from the assumption that the socioeconomic context could influence the indices of presence and permanence of banditry. Finally, the identification of the sheaves and bandits that took place in the State of Michoacán between 1877-1893 is carried out, this, to delimit the morphology of the banditry and the relationship it had with the context that was being lived in Mexican society.
\end{abstract}

Keywords: Banditry, Bandits, sheaves, porfiriato socioeconomic

*xyzpao@hotmail.com 


\section{EL BANDOLERISMO MEXICANO EN LA HISTORIOGRAFÍA}

El tema del bandolerismo en México cuenta con una amplia tradición académica, entre los resultados se precisa que dicha práctica social fue cambiando tanto en forma como en sentido, lo mismo había bandidos que respondían a situaciones económicas, que a cuestiones como la guerra o la política, incluso, hubo quienes gozaron de popularidad. En las investigaciones también se puntualiza que el surgimiento del bandidaje respondió a múltiples factores, por lo que su interpretación resulta compleja, ya que, no se explica únicamente por los atributos personales de los sujetos, debemos interpretarlo tomando en cuenta las circunstancias históricas (Verdugo, 1991). Si bien se han ubicado bandidos sociales también se han comprobado otros tipos, incluso más recurrentes, como el bandolerismo empresarial o político. Por ello, esbozamos que el bandidaje mexicano pasó por tres momentos desde la independencia hasta el fin del XIX:

1) En los ańos previos y principalmente durante la independencia, los bandidos actuaron bajo la etiqueta de patriotas y casi de manera general se unieron al mejor postor, sin necesariamente contar con una convicción ideológica concreta, si la tenían, les venía de fuera de su realidad inmediata por medio de grupos o sujetos.

2) En la primera mitad del siglo XIX y hacia el fin de la década de los sesenta el bandolerismo logró la fuerza necesaria para ser una práctica en beneficio de solo unos cuantos y no necesariamente de un movimiento, con lo que su carácter social es casi nulo, pues sus acciones pudieron estar sumidas en deseos de terceros.

3) Finalmente, particularmente durante el Porfiriato se denotaría un cambio de modalidad en el bandolerismo, principalmente, debido a que no se presentaron grandes luchas internas ni intervenciones extranjeras, las problemáticas del contexto giraron en torno a cuestiones regionales y el bandidaje sería la respuesta a la inmediatez de las circunstancias socioeconómicas.

Para el primero de los momentos se ha identificado que fue muy poca la práctica del bandolerismo social. Las obras de William Taylor (1980), Eric Van Young (2009) y Christon Archer (1982) son parte 
fundamental de lo que se conoce hasta el momento, entre otras cosas, confirman la complejidad del tema en el caso mexicano. Sus estudios pusieron a discusión cuál fue el tipo de bandidaje que se desarrolló a fines de la época colonial y durante la lucha independentista, evidenciando que no solo fue social, incluso, propusieron otras modalidades iguales o más practicadas, William Taylor (1990: 195) menciona que "pocas veces es posible distinguir con claridad en las gavillas de la independencia movimientos políticos, bandidos sociales y delincuentes comunes" La presencia del bandidaje en los casos regionales ha demostrado una práctica social con significativo impacto en la construcción del entonces naciente Estado mexicano.

La relación de los bandidos con las estructuras y las gentes de poder fue fundamental en ese primer momento, en gran medida porque los bandidos actuaban con cierto grado de protección de distintas gentes o sectores. Por lo tanto, quienes recurrieron al bandidaje fue mayormente como un instrumento político para adquirir relativa legitimidad. Con base en ello, se propuso el bandido guerrillero, bajo el cual se englobaría a quienes se conducían bajo la bandera de patriotismo, pero en sus acciones cometían abusos que afectaban por igual a la sociedad y no únicamente a alguna facción política. El movimiento independentista obtuvo ventajas con la presencia de los bandidos, pero, estos no siempre exhibían clara bandera política e incluso cambiaban sus intereses según contextos. En el caso de las investigación centradas en este primer momento, se coincide con la afirmación referente a que en comparación con América Latina, las "clases inferiores participaron en estos conflictos como clientela o como aliados de las elites; a veces se emanciparon y pelearon por su cuenta" (Katz, 2004: 459).

Tras la independencia mexicana se considera que se desplegó un segundo modelo de bandidaje, ello directamente relacionado con el siglo XIX mexicano, en sumo conflictivo, basta hacer mención de que entre 1820-1900 hubo 102 revueltas (Coatsworth, 1990: 37), lo que haría de tal práctica una de las "responsables de la continuación de la violencia" (Vanderwood, 1984: 47). El bandolerismo lograría un aumento regional considerable entre esos años, teniendo una amplia 
connotación regional en diversas gavillas. La lucha de independencia favoreció la presencia de aspectos que resultaban idóneos para el posterior ejercicio del bandidaje: la posesión de armas por parte de sujetos particulares, relativos conocimientos militares, aunado a que no todos se vieron beneficiados con los resultados.

El ejercicio del bandolerismo llegó a niveles considerables entre 1821-1870, la evidencia de ello fue que se legislaron diversas leyes específicas y de carácter federal para su combate, aunque, dicha práctica continuaría a lo largo del XIX (Solares, 1999a). El bandidaje fue relacionado en este segundo momento con términos como salteador de caminos, gavilleros, forasteros, malhechores, ladrones de caminos y las connotaciones que implicaban. Los trabajos de Laura Solares (1999a), Leticia Reina (1980), Jaime Olveda (2003) son la plataforma para los interesados en dicha temporalidad, resaltándose la importancia del contexto histórico por las diversas y extensas pugnas políticas, intervenciones extranjeras, guerras civiles, crisis económicas y agrícolas que favorecían su presencia y permanencia, se ha particularizado que en dicho periodo el bandidaje fue atacado mediante acciones concretas pero sin logros, pues no existía la estabilidad política y económica.

Laura Solares señala que representó un tipo de rebelión individual o colectiva, presente la mayoría de las veces en contextos de incertidumbre política y económica, agudizado en los espacios rurales; en la Reforma adoptó tantos y tan variados matices que merece un estudio pormenorizado; durante la intervención francesa se trataba por igual a "dispersos como intervencionistas, el título fue por el tipo de acciones que ejercían, incluso el plagio de hombres, mujeres y niños" (1999a: 19). Sobre la forma en que era percibido no se niega que posiblemente pudieron en ocasiones mezclarse los factores que daban origen, podría iniciar como bandidaje social pero a la postre fungir como un acto de resistencia.

En una gran parte de las investigaciones se considera que el bandolerismo entre 1821-1870 rebasaría "los límites de la atención que el estado podía dispensarle, por ello, lo usual era que gozasen de total impunidad en las áreas rurales, atreviéndose incluso a atacar las 
ciudades e incluso el no respetar la condición social de sus víctimas resultaba un denominador común al resto de los maleantes" (Solares, 1999b: 38-39). Debido a las circunstancias del contexto histórico, se particulariza que recurrieron al bandolerismo distintos sectores de la sociedad, entre otros, los campesinos que habían perdido tierras e incluso militares que después de concluidas las luchas no tenían cabida o eran desertores. A los mencionados se sumarian de manera indirecta representantes de las antiguas autoridades civiles y militares, comerciantes, hacendados, rancheros, mineros, etc. quienes avalarían sus acciones e incluso pactarían.

La inclusión de tan diversos actores demuestra la diversificación de los motivos, formas, acciones e intereses del bandolerismo. Respecto a las motivantes que se tenían se pueden dividir en las que fueron producto de circunstancias históricas como el desempleo, la desigualdad económica o la pobreza, el alza de precios de los productos alimenticios, los costos de las mercancías de uso diario, etc. A las cuales se sumarían las que eran resultado de las políticas implementadas por los grupos en el poder como el aumento de impuestos, las contribuciones para apoyar al gobierno, etc. En el caso mexicano tales condiciones no únicamente aplicaban para el bandidaje sino también para la presencia de otras prácticas sociales (Reina, 1993b), lo que en cierta medida explica la relación de las condiciones del contexto con el bandidaje entre 1821-1870. No es de extrañar que los bandidos se catalogaran como individualistas en sus acciones, puesto que en no pocas ocasiones se aliaron con terceros para aprovecharse de las condiciones socioeconómicas del momento (Barreto, 2007), mientras que Vanderwood señala que durante como después de la independencia

Habían saqueado en calidad de monárquicos como republicanos y cuando terminó no quisieron regresar a sus hogares, tenían la intención de tratar con los nuevos dueńos del poder y cuando no tuvieron para pagarles se volvieron bandoleros y se hicieron servidores de los intereses regionales (1984: 47). 
En lo que concierne al tercer momento del bandolerismo entre 1870-1910, se puede iniciar señalando que el país estaría inmerso en un proceso de modernización institucional y de replanteamiento económico y social, sobre lo cual se ha precisado que "El México de la modernización porfirista es en gran parte un país de modernización preindustrial, inducida indudablemente por el comercio y los polos exteriores, pero resultante también de la lógica interna de la antigua sociedad" (Guerra, 1991: 352). No es que antes no se pretendieran tales acciones, sino que en el régimen porfirista (1877-1911) fueron una prioridad. Los efectos de las acciones que fueron impulsadas durante el régimen porfirista influyeron en el desarrollo del bandidaje, puesto que efectivamente se ampliaron las capacidades del estado, llegando al grado de que, tal como puntualizó Medina Peña, "casi todo es tolerable siempre y cuando no se trasponga un límite: incursionar en las vías de hecho, alentar o incurrir en la sedición y la revuelta" (Medina, 2004: 75).

Dicho interés del gobierno porfirista en el combate al bandidaje fue debido a que "por sí mismo echa abajo la imagen de paz y consenso nacional, tan vital para un proyecto nacional" (Falcón, 1998: 387) siendo por lo tanto, una preocupación que afectaba a múltiples sectores sociales e institucionales, de allí que todo aquel sujeto "que se pronunciara contra el gobierno pasaba a engrosar las filas de los hombres al margen de la ley y eran tachados de revoltoso, gavillero y bandido" (Gantus, 2008: 51). Los estudios de Trujillo (2010), Aparecida (2001), Verdugo (1992), abordan esta tercer temporalidad, los cuales se han orientado más hacia la tesis de un bandidaje centrado en lugares solitarios, individualistas y dispersos en bandas pequeñas, en relación con fuerzas militares e incluso pasaban a ser rurales, uso recurrente de la violencia y no se distinguía el origen social del perjudicado. En el proyecto porfirista la paz y progresiva eliminación de los bandidos era un objetivo central, sin embargo, recientemente también se ha evidenciado que

El bandido de tiempo completo en el Porfiriato fue un mito, estos eran casados, con vivienda, oficio, vinculados 
a la tierra, sus acciones individuales, la formación de partidas armadas perecía estar más limitada a los asaltos a la infraestructura ferrocarrilera o minera y al abigeato o al servicio de terratenientes...formaban parte de distintas esferas de la economía, su supervivencia diaria no dependía de la actividad delictiva (Aparecida, 2005: 167, 179).

En la prensa porfirista se localizaron opiniones que demuestran lo preocupante del bandidaje, en 1877 se aludía que “se está haciendo endémico y es una enfermedad social" (La Colonia española, 6 de febrero de 1877: 1-2), mientras que hacia 1880 "la seguridad de los caminos y los campos es una de las necesidades más apremiantes" (El Nacional, 11 de julio de 1880, p. 1), finalmente, en 1887 "dos cosas han sido el oprobio por muchos años, el bandidaje en caminos y el de los pronunciamientos" (El Diario del Hogar, 30 de noviembre de 1887). Asimismo, se han identificado características distintas a las de los momentos referidos con anterioridad, la "aparición de un número reducido de bandoleros solitarios, robos más específicos, pandillas más pequeńas, corta pervivencia y uso regular de la violencia" (Vanderwood, 1984: 55), ello ha llevado a que se precise que tuvo un matiz diferente, resultado principalmente del fortalecimiento del Estado y sus medios coactivos (Vanderwood, 1994: 127-130).

Con respecto al nivel de preocupación social que significaba el bandolerismo, la prensa también es de suma utilidad, se refería entre otras cosas que "es el mayor y más atendible de nuestros males porque altera el orden y la tranquilidad pública” (El Nacional, 11 de julio de 1880) y "se está haciendo endémico" (La colonia española, 10 de febrero de 1877), además se "ha desarrollado de manera estupenda" (El siglo XIX, 4 de mayo de 1886). La particular atención que se prestó al bandidaje durante el régimen porfirista, se vio fortalecida porque que durante un tiempo el gobierno federal no se preocupó "porque estallaran revueltas en gran escala, guerras de castas o fuera derribado por un levantamiento generalizado" (Dale, 1986: p. 17), por lo que prácticas sociales como el bandidaje lograron atención 
significativa e incluso particular por parte de la autoridad a pesar de no tener las dimensiones ni capacidades anteriores.

Antes de proseguir con el análisis del bandolerismo en una región mexicana, que es la propuesta central de esta investigación, es pertinente puntualizar que la historiografía ha planteado que dicha práctica pudo constituir entre otras situaciones, una "forma de protesta campesina que gira en torno a elementos de clase que guarda íntima relación con las resistencias y los levantamientos" y que la realidad de los bandidos estaba vinculada con "individuos perseguidos por la justicia, capaces de robar y matar sin distinción". En nuestro caso, planteamos la presencia de un bandidaje afín con aquellos "modos de reacción más individuales, aunque originados en un medio de experiencia colectiva y estatus inferior" (Buve, 1971: 453).

Es decir, si bien era un acto colectivo es notorio que en el espacio y tiempo de estudio el bandolerismo guardó cierta distancia de las prácticas sociales con pretensiones más amplias, las cuales en gran medida eran consecuencia de la interacción de sujetos con anhelos y posiciones distintas. Por lo tanto, planteamos que el bandolerismo si bien fue una acción en torno a la cual se agrupaban varios individuos, ello no le hacía necesariamente un acto colectivo con propuestas únicas ni ideologías políticas amplias que pretendieran trastocar el orden gubernamental, más bien, fungió como un medio ordinario para una serie de problemas o necesidades cotidianas que atañían a un grupo de sujetos con similitudes personales como el desempleo, la falta de ingresos, el aumento de precios, las sequias, etc.

Por lo tanto, pretendemos demostrar que el bandolerismo entre 1877-1893 fue una práctica rural ejercida por sujetos afines estrechamente con el contexto regional, siendo un medio por el cual se buscaba la satisfacción de aspectos delimitados a un núcleo inmediato como la familia o factores socioeconómicos ordinarios. $\mathrm{Al}$ respecto Taylor (1987) a lo largo de su investigación localizó que para Jalisco hubo ladrones de la variedad más doméstica que robaban para su beneficio personal. La propuesta de Richard Slatta resulta pertinente, porque le otorga un sentido utilitario y moldeable de 
por el reconocimiento de la coexistencia de intereses específicos, al precisar que "Banditry can be viewed as one of many strategies used by the to adaptto constraints and changes imposed from above" (1987: 172). Mientras que lo referido por Vanderwood sobre el bandido es de sumo valor, al reconocer causas que lo alejan de la tradicional protesta campesina y no lo delimita a la lucha contra de las autoridades e instituciones, diversifica su sentido como

Aquel individuo y sus secuaces que perseguían su propio interés, que se hallaban excluidos de las posibilidades y oportunidades y no digamos los beneficios de la sociedad en general y que fomentaban el desorden a manera de palanca para entrar en un sistema reservado (Vanderwood, 1986: 11).

Con respecto al término gavilla, calificativo designado en la época por las autoridades mexicanas al grupo de individuos que cometían bandolerismo a lo largo del siglo XIX. En 1877 se precisaba que había una recurrente presencia de "gavillas de malhechores" (Archivo Histórico de la Secretaria de la Defensa Nacional (en adelante AHSDN), Siglo XIX, Fondo Michoacán, exp.481.4/11952), se añadía que todos aquellos individuos que anden en despoblado, en las calles, los caminos o campos se les tendrán por sospechosos, mientras que en un decreto de 1886 se enunciaba que para evitar las acciones de los bandidos en gavilla se apliquen las medidas necesarias (AHSDN, Siglo XIX, Fondo Michoacán, XI/481.4/12249).

El gobierno encabezado por Porfirio Díaz desde su primer mandato inició una amplia empresa para erradicar el bandolerismo, las opiniones sobre los resultados son ambiguas, para algunos "el principal beneficio del régimen es el restablecimiento de la paz y la progresiva eliminación de los bandidos" (Guerra, 1991: 325) mientras que otros solo reconocían "la paz, más no la seguridad pública” (Falcón, 1998: 388). En las diversas regiones mexicanas variaría la presencia y permanencia del bandidaje, así como, los niveles de aceptación e implementación de las acciones para combatirle, por lo que la empresa de afrontar dicha práctica fue compleja. 
EL BANDOLERISMO EN UNA REGIÓN PORFIRISTA: EL BAJÍO MEXICANO El interés por erradicar el bandolerismo en México encontraría particular aceptación desde el inicio del régimen porfirista en uno de los estados que conforman el país, el estado de Michoacán. Durante la gubernatura interina de Manuel González en 1877, se realizó una férrea campaña militar y legal en contra de la inseguridad estatal, específicamente contra dicha práctica social. Al hacerse cargo del ejecutivo solicitaba a los michoacanos que "ayuden a conseguir el afianzamiento de la paz" y este mismo personaje reconocía que la administración de justicia "merece atención seria" (El libre Sufragio, 5 de febrero de 1880), situación similar aludía en 1886 otro gobernador, Mariano Jiménez, al decir que "toca iniciar una época de paz y progreso" por "todos lados hay malhechores" (El siglo XIX, 28 de septiembre de 1886).

El Estado de Michoacán forma parte de una región más amplia conocida como el Bajío mexicano, que se integra con porciones territoriales de Guanajuato, Jalisco, Querétaro y Michoacán. El Bajío desde mediados del siglo XVIII iniciaría un progreso que facilitaría su consolidación como una de las regiones más importantes de México. Tal apogeo era visible en la economía y la demografía, con base en que se viviría "la combinación de urbanización, industria textil, minería y agricultura” (Brading, 1991: 303). La producción agropecuaria beneficiaba la existencia de un mercado considerable y un constante intercambio comercial. El movimiento regular de alimentos, dinero, animales, mercancías e individuos, propiciaba una constante circulación mercantil y financiera, al respecto Luis González señaló:

La explosión económica aventajó a la demográfica y urbanística, rara vez por la aventura en nuevos negocios, casi siempre por el desarrollo veloz de las tareas tradicionales... tan prospera que le acarreó el título de granero de la Nueva España...se hizo de una red caminera caminada por recuas de mulas y transportes de mayor fuste y ruido como los carros que rodaban por los caminos mayores de los reales de minas... 
es un lugar común la función abastecedora de carnes, granos y manufacturas...lo distintivo de la vida regional consistió en el uso desmedido del caballo (González, 1989: 104-106).

A inicios de la década de 1870 se decía que en Michoacán “tan ingente es la fertilidad del terreno que en varias regiones sin el auxilio del abono, el suelo produce quinientas fanegas por una de maíz y cincuenta de trigo" (Ruiz, 1872: 324); en 1895 la producción era de "la más alta importancia, es uno de los mayores productores" $\left(\mathrm{O}^{\prime}\right.$ Farril, 1895: 172), hacia 1899 "por la riqueza agrícola es el primer Estado cuyas producciones anuales sobrepasan los 30 millones de pesos, la que ningún otro" (Figueroa, 1899: 334). Al respecto, cabe puntualizar que, en el año de 1890 se refería que también el Bajío "por años ha prestado elementos a los trastornadores, entre ellos el económico" (La Patria, 22 de mayo de 1890: 2).

En lo que concierne a algunas de las ciudades y lugares que conformaban el estado de Michoacán, se ha localizado que gozaban de buenas características en su productividad. En el caso de Zamora empezó a definirse como centro agrícola-comercial por "la fertilidad delvalle, la extensión del riego y la diversidad de inversión" (Gutiérrez, 1986: 271); en La Piedad las cosechas son casi seguras y podían estimarse en siete millones de fanegas anuales; el ganado vacuno es el mejor del centro; en Jiquilpan "la cantidad de producción es similar con los lugares cercanos"; finalmente Puruándiro, "centro agrícola-comercial, escenario medular de la economía local, la tercera ciudad del estado" (García, 2010b: 229). Tenemos claro que el bandolerismo no sólo existió y era fructífero en donde se vivía una movilidad financiera y comercial, pero sin duda, un cuadro económico como el descrito debió de ser un significativo foco de atracción para quienes notaron en el bandolerismo una forma de subsistir o emplearse de manera temporal.

Los aspectos económicos casi uniformes y considerables del Bajío michoacano propiciaban una mayor posibilidad de éxito para los bandidos, entre otras cosas porque su producción era mayor que en otros lugares. En Puruándiro la cosecha anual "era de 120 
mil fanegas de maíz y 3, 400 cargas de trigo y era el escenario del tráfico mercantil de la región", aunado a que existían "parte de las principales haciendas cerealeras y sus localidades estaban en un importante corredor que veía desfilar hombres, animales, carretas, mercancías y dinero" (Sánchez, 1989: 238). William Taylor relaciona la proliferación del bandidaje en Jalisco a "cambios sustanciales en las condiciones materiales de la región como el crecimiento de la población, la agricultura comercial y la presión sobre la tierra" (Taylor, 1990: 203), aspectos también perceptibles en el Bajío michoacano.

La dinámica comercial porfiriana de los caminos de una parte de Michoacán representaba la existencia de significativas rutas de intercambio entre ellos y con otros estados, puesto que estaban colocados útilmente para enviar a todas direcciones (Ruiz, 1872: 824826). Tal situación representó un atractivo tanto por las cantidades de productos que circulaban como por el número de individuos que transitaban constantemente. Parte de su importancia se encuentra en distintas publicaciones de la época, las cuales refieren entre otras cosas que

El camino principal iba a Zamora, pasando por la hacienda de Guaracha y el pueblo de Guarachita...otro salía a Sahuayo por la calera. Y si bien la ciudad quedó fuera de la vía ZamoraLos Reyes en 1900, no estaba lejos de la estación Moreno de Guaracha, a la que se llegaba por diligencia; quienes se embarcaban en La Palma para tomar tren a Ocotlán (Ochoa, 2010: 120).

El de La Piedad era un cardinal enclave comercial al tener estación de tren, que comunicaba al interior del estado y hacia el exterior

Para estos ańos se veía transitar a los arrieros con sus mulas, sobre todo en el camino Zamora-La Piedad que era uno de los conductos más importantes para dar salida a los productos michoacanos hacia el norte (García, 2010a: 135). 
Ante tan halagador panorama se oponían los semblantes sociales de los michoacanos, los cuales creemos relevantes para poder explicar los motivos de los bandidos. Es pertinente hacer mención de la afinidad que consideramos existe con la propuesta referente a que "bastan los agravantes que padece una población, ni los intereses de clase que pueden converger en un momento para explicar sus características" (Rangel, 2006: 17). En este punto es necesario referir las opiniones sobre la realidad social que se vivía, si bien aludimos las buenas condiciones de la producción del espacio de estudio, estas contrastan con el bienestar social de la población, coyuntura por la cual el bandidaje encontraría presencia.

En Puruándiro la "infraestructura era raquítica e insuficiente desde el punto de vista de las comunicaciones" aunado a "una remarcada diferencia de clases" (Ortiz, 1980: 200), recurrentes abusos a los sectores populares, desigual reparto de tierras, constante aumento poblacional y marcada concentración de la riqueza. Mientras que en Jiquilpan "pese a la modernidad material la sociedad era poco igualitaria” (Ochoa, 2010: 120) y en Puruándiro "no obstante las mejoras la mayoría de la población permaneció al margen del avance y en todo caso eso solamente coadyuvó a remarcar todavía más las diferencias de clase...el periodo de paz y progreso estaba lejos" (García, 2010b: 232).

Sobre la presencia del bandidaje a lo largo del Bajío michoacano ubicamos algunos comentarios de la época en los cuales se decía que el viaje de los pasajeros incluía "el temor de ser asaltados por bandidos" (Ortiz, 1980: 197), debido a que la seguridad "está en pésimas condiciones" y porque en unos sitios "existía una vieja tradición de asaltos" (El Monitor republicano, 19 de agosto de 1881). En Zamora se exponía por las autoridades que "es uno de los que más debe vigilarse por sus antecedentes" (Archivo Histórico Casa Morelos (en adelante AHCM), Gobierno, Policía y Guerra, Movimiento de fuerzas, Caja 264, Exp. 1). En el año de 1879 la situación no había cambiado pues se precisaba que el bandidaje constituía "uno de los delitos de mayor presencia y difícil averiguación porque se aprovecha la soledad de los caminos" y en La 
Piedad la paz y el progreso estaban lejos de ser (García, 2010a: 134139).

Los propios habitantes de las poblaciones dejaron testimonios de la inseguridad, por lo que el bandidaje tuvo poca aceptación entre los sectores pobres, puesto que se localizaron documentos a nombre de trabajadores del campo o de habitantes de los pueblos o tenencias. Ello indica los niveles de inquietud y preocupación entre aquellos que no formaban parte de los grandes intereses económicos o políticos como los rancheros, comerciantes, políticos y hacendados, quienes por lo regular tenían su propia defensa o contaban con fuerzas militares fijas como en Urundaneo y Villachuato (AHSDN, Siglo XIX, Michoacán, Exp. 481.4/12361).

Asimismo, se refiere que "los vecinos de las haciendas y rancherías han ofrecido continuar persecuciones contra las diversas gavillas" (AHSDN, Siglo XIX, Michoacán, Exp. 481.4/11958), incluso, hacen peticiones para que las fuerzas no se fueran por "la gran inseguridad" (AHCM, Gobierno, Policía y Guerra, Movimiento de fuerzas, Caja 264, Exp. 3) y donde estas no había se solicitaban, sino llegaban se organizaban como sucedió en Huango donde estaban "dispuestos a defenderse por la inseguridad". Ello debió influir para que se cambiara infantería por caballería en 1878 y asignaran destacamentos permanentes. Incluso se proponía que "para conseguir mejores resultados es forzoso reformar la legislación penal, haciéndola más severa para el asalto" (Memoria, 1892-1894: 28).

En diversas investigaciones se ha puntualizado que "las actividades delictivas se definen más por sus características externas que por sus elementos inherentes...su intensidad, duración y cariz dependen del contexto" (Aparecida, 2005: 346), esto, con base en que "las crisis, la economía, la geografía, la miseria, la tradición y la oportunidad, todo tiene un papel" (Vanderwood, 1986: 30). Tales ideas amplían el margen de análisis del bandolerismo, porque refieren una práctica social en cierta medida independiente de otras, ya que se reconoce que quienes participaban tenían intereses que iban más allá de algún sentimiento personal como la venganza o la inquietud política. 
Al realizar el análisis de los afectados por las 62 gavillas localizadas entre 1877-1893, se puede denotar el distanciamiento con facciones o movimientos políticos, no así con lo socioeconómico. En primer lugar se hallaban aquellos sujetos que fueron robados y que no tenían recursos económicos o mercantiles mayores y que fueron robos en sitios abiertos, en este caso 45 gavillas: nos referimos al campesino común, jornalero, artesano, arriero, etc. En segundo, los que tenían algún tipo de bien material como los hacendados, comerciantes y rancheros de regular importancia local, a los cuales de manera casi común se les hurtó en espacios fijos como sus propiedades con 15 gavillas. Por último, algún tipo de autoridad civil o militar solo en dos, en ningún caso se encontró que realizaran daños a edificios públicos ni que la vida de algún representante del poder corriera más peligro que cualquier otro sujeto.

El bandidaje michoacano en buena medida consideramos que fue producto de la dinámica local y por lo tanto no es una práctica endémica, aunque si una respuesta local, ello es notorio al analizar que los ańos en que se cometieron los robos, los cuales se relacionan con sucesos locales específicos, particularmente sociales y económicos. El distanciarlo de lo político es porque solo en una ocasión se pudiera relacionar con un suceso de tal naturaleza, las elecciones de 1880-1881, aunque por la morfología e integrantes de las gavillas es poca o nula la relación con las cuestiones políticas, eran gavillas en un número pequeńo, sus acciones fueron en sitios lejanos a donde fueron las elecciones y robaron maíz y dinero.

\section{El bandidaje en Michoacán: Respuesta al contexto} SOCIOECONÓMICO

Del análisis de las 62 gavillas resalta como una práctica social sumamente local en sus áreas de acción, debido a que 60 bandas realizaron sus robos en el interior de Michoacán, una en el estado de Guanajuato y otra en el de Jalisco, a lo que se suma que la residencia y vecindad de 85 de los 89 bandidos estaba en Michoacán, tres en Guanajuato y uno de Jalisco. Los datos indican el poco o nulo vínculo con dificultades e intereses ajenos a los estatales, las 
condiciones económicas y sociales son notorias como uno de los problemas centrales, los ańos de mayor y menor actividad guardan relación con sucesos ocurridos en el Bajío michoacano, entre ellos: escases de alimentos y crisis económica de 1877-1878; aumento de los precios de productos como el maíz y el trigo en 1880-1881; incremento de impuestos de 1884-1885; los problemas naturales que crearon escases y alza de precios entre 1890-1892.

La falta de empleo y dinero fue reconocida por la prensa, debido a que se refieren entre otras situaciones como que "la miseria es muy mala consejera” (El siglo XIX, 14 de octubre de 1878). Cabe precisar que no dudamos que cuando se presentó la oportunidad de robar objetos no necesarios se llevó a cabo, ubicamos tres personajes: Marcial Bravo, Joaquín Núñez y Juan Mora. Al analizar los objetos robados se identificó la búsqueda por repararse privaciones bastante específicas, particularmente porque en su mayoría comprendían bienes cotidianos y en cantidades no tan amplias. Por lo tanto, no creemos que tuviera fines empresariales o ideológicos y menos que gozara de protectores, es decir, su fin era la autosuficiencia atańida con lo socioeconómico, con base en que

Las circunstancias estructurales, como la pobreza provocada o agudizada por el desempleo y la falta de riqueza propiciaban que ciertos grupos de la población se hicieran de ocupaciones alternativas (Magallanes, 2008: 58).

Por lo tanto, el bandidaje fue una práctica delimitada en buena medida a lo local por conveniencia, ventaja y por limitantes e incapacidades gubernamentales, por lo que los bandidos michoacanos podrían entrar en aquellos "sujetos capaces de definir y elegir distintas estrategias que aparecen siempre como creativas, reversibles y calculadas" (Trujillo, 2010: 32). Lo anterior implica que el bandidaje estuvo estrechamente relacionado con las condiciones del estado y no de algún proyecto mayor. Lo pensamos como un medio al que recurrieron sujetos con similitudes en las condiciones que vivían y no más allá de lo cercano. 
El bandidaje michoacano no tuvo ni la magnitud ni la capacidad de épocas previas según los robos cometidos, debido a que los afectados no eran aquellos que tenían amplias posibilidades económicas o políticas y los objetos robados eran bastante definidos y en cantidades no amplias. Es decir, eran bandas que sabían de sus capacidades porque se limitaban a lo necesario y en escenarios bastante determinados, aunque, ello no es indicativo de actos mínimos sino la existencia de una práctica circunscrita (de manera premeditada o forzada) a gavillas pequeńas con causas-fines cortos y específicos, por lo que, "la visión de los actores, sin que fuese limitada, se circunscribía a fuerzas que podían controlar y manipular" (Rangel, 2006: 21).

La relación primeramente entre el año de surgimiento y el contexto regional y en segundo entre los meses de robo con ciertas etapas del año, hacen evidentes algunos de los objetivos que se tenían con la formación de una gavilla y muestran parte del carácter del bandolerismo en la región de estudio. De acuerdo con ambos aspectos, esbozamos la existencia de cierta planeación en el ejercicio del bandidaje, debido a que su recurrencia era mayor en ciertos momentos del año, por ello, concordamos en que "estos estilos de vida eran asumidos como transitorios...por lo que los bandidos estaban conscientes de que llegaría el momento de integrarse al orden" (Padilla, 2010: 187).

La mayor actividad fue en los meses cercanos a la siembra e incluso durante, como en abril y mayo cuando el producto comenzaba a escasear y aumentaba el desempleo; mientras que en octubre estaba por iniciar la cosecha y aumentaba el número de quienes no fueron contratados. Por último, en diciembre y enero terminaba la cosecha, el empleo escaseaba, se vivía la falta de dinero y el alza en la circulación de bienes y personas por los caminos. Los meses se relacionan directamente con períodos del año bastante concretos, como los previos o posteriores a la siembra y cosecha o con las temporadas de secas o lluvias. Al respecto Vanderwood precisó que "la naturaleza temporalera de la economía rural contribuía" (1986: 31 , por lo que tales aspectos llevan a suponer que el bandidaje fue 
una manera de resistir pero sin la intención de perturbar el orden, sino de poder afrontar la cotidianidad.

El analizar lo robado creemos nos acercará en cierta medida al carácter de las gavillas, cabe precisar que tenemos claro que tal dato es complejo de resolver, puesto que un robo se componía de distintos objetos, pero, en la mayoría de los casos es evidente la tendencia hacia ciertos bienes. Tal aspecto se tiene como una constante en diversos estudios, lo que ha llevado a que se afirme que "se roba lo que se ansia o está sujeto a comercio... se roba lo que preferentemente se consume" (Camino, 2010: 118). Lo anterior resulta un tanto generalizador, pero no es así, en un análisis detenido es clara la presencia de lo socioeconómico: eran objetos ordinarios y necesarios en la vida de un sujeto o una familia y ciertamente comercializables. Sin embargo, se ha probado que hubo acaparamiento y desastres naturales que obligaron a que los sujetos se vieran en la necesidad de poseer dinero para adquirirlos, de lo cual se deriva parte de la explicación de que fueran lo más robado.

El hecho de que el dinero y los animales fueran lo más robado creemos tenía relación estrecha con la región del Bajío michoacano, debido a que al ser un espacio agrícola de amplia producción, era poco probable que los productos de tal actividad fueran tan escasos como en las regiones sin esa característica. Sin embargo, también era una constante despojar de productos agrícolas como el maíz, trigo o frijol. Sobre estos cabe precisar que eran más robados en los meses de escases o cuando se les imponían impuestos como sucedió con el maíz. Por lo tanto, la sustracción de frutos agrarios respondía a una lógica cotidiana como la alimentación, más, si se tenía familia como gran parte de los bandidos, en este caso 60 de 89 sujetos.

El despojo de armas y ropa especulamos era un tanto circunstancial, es decir, no eran objetivos específicos y con su despojo solo se procuraba aumentar la ganancia, ello, debido a que su uso y venta resultaba más compleja, tanto porque las armas estaban prohibidas sin permisos como porque la ropa al no ser tan accesible por la poca producción y costo era fácil reconocerla por los afectados. Es conveniente precisar que fue un hecho que prevalecieron los robos 
en especie, tanto de maíz como de trigo, frijol y cierto tipo de animales, aunque también de armas, sombreros, hachas, machetes y reatas, aunque fueron los menos.

Un aspecto relacionado con lo anterior son las cantidades robadas y su valor. Esto ha sido difícil de aclarar porque los bandidos declaraban una cosa y los afectados otra, por ello, para este aspecto tomaremos solamente 30 avalúos que hicieron sujetos particulares en distintos ańos. Las cantidades variaron pero se pueden ubicar tres lapsos temporales: en el primero se superan los 100 pesos por productos diversos; en el segundo se reducen fluctuando los 30-60 pesos con artículos más concretos; en el tercero aumentan pasando los 60 pesos por productos relacionados a granos y animales. Por lo tanto, con base en el tipo de productos no se puede vincular a las gavillas con movimientos sociales amplios, al contrario, eran bienes o mercancías que ciertamente facilitaban dinero inmediato y reparaban necesidades cotidianas como la alimentación o falta de recursos para comprar lo faltante en la vida diaria de una familia porfirista.

Al compararse el salario de los jornaleros, arrieros y labradores (que componían el grueso de las gavillas) con el valor de algunos de los productos básicos en la vida cotidiana del Porfiriato, resulta evidente que el botín era redituable para sortear únicamente una dificultad momentánea y no tan amplia. Ello por tres aspectos: el primero es que el salario entre 1877-1893 osciló entre los 25-40 centavos por día, poco en relación con el promedio de la ganancia del robo; el segundo es que la mayoría de lo robado era relativamente fácil de comerciar porque eran productos de uso o consumo regular; el tercero es que con lo obtenido se podían adquirir productos básicos, debido a que la fanega de maíz costaba entre 10 y 15 reales, la de frijol 4 pesos y la de garbanzo 15 reales y la carga de trigo 4-6 pesos (Memoria, 1883).

$\mathrm{Al}$ respecto, la cantidad de miembros de las bandas oscilaba entre los 40 y los tres sujetos, siendo el promedio 15 . Tal cifra no es amplia y en parte explica que las acciones estuvieran circunscritas a zonas e individuos determinados. Es un hecho que a lo largo del tiempo de 
nuestra investigación tuvieron distintas cantidades de integrantes: fueron más grandes en el primer gobierno de Porfirio Díaz 1877 1880, quizá porque en ańos anteriores eran gavillas más grandes; siendo de menor cantidad cuando se vivió un momento económico regular y por la creación y ampliación de leyes e instancias judiciales como fue a partir de 1881 y hasta 1890 . Aumentando nuevamente entre los ańos de 1890-1893 por los distintas crisis naturales.

\begin{tabular}{|c|c|c|c|}
\hline AÑO & $\begin{array}{c}\text { BANDIDOS } \\
\text { POR } \\
\text { GAVILLA } \\
\end{array}$ & OBJETOS & $\begin{array}{c}\text { VALOR } \\
\text { DE LO } \\
\text { ROBADO }\end{array}$ \\
\hline 1877 & 15 & Cuanto podían & 150 pesos \\
\hline 1877 & 6 & 1 sombrero, dinero y 1 chivo & 35 pesos \\
\hline 1877 & 18 & Hachas, burros, frazadas & 102 pesos \\
\hline 1878 & 6 & 5 Burros, 2 cargas de costales, 3 frazadas, hacha & 60 pesos \\
\hline 1878 & 10 & Una yegua, dinero y 2 reatas & 38 pesos \\
\hline 1878 & 15 & Maíz, trigo y dinero & 22 pesos \\
\hline 1879 & 3 & Burra con leña & 25 pesos \\
\hline 1879 & 3 & Dinero & 20 pesos \\
\hline 1879 & 16 & 6 burros, una camisa y maíz & 96 pesos \\
\hline 1880 & 10 & Artículos diversos & 128 pesos \\
\hline 1880 & 8 & Leńa y dinero & 32 pesos \\
\hline 1880 & 13 & Maíz y dinero & 24 pesos \\
\hline 1881 & 4 & Dinero y sombrero & 16 pesos \\
\hline 1881 & 8 & Dinero y alimentos & 25 pesos \\
\hline 1881 & 3 & Maíz y una frazada & 5 pesos \\
\hline 1883 & 3 & 3 mulas y un caballo & 55 pesos \\
\hline 1884 & 5 & Dinero y reata & 5 pesos \\
\hline 1887 & 4 & 9 bueyes & 217 pesos \\
\hline 1887 & 5 & Dinero y leña & 23 pesos \\
\hline 1888 & 7 & Dinero y alimentos & 23 pesos \\
\hline 1889 & 5 & Maíz, dinero y ropa & 35 pesos \\
\hline 1892 & 3 & 2 zarapes, pistola y media fanega de maíz & 45 pesos \\
\hline 1892 & 5 & Dinero & 70 pesos \\
\hline 1892 & 2 & Ropa y maíz & 51 pesos \\
\hline 1892 & 15 & Dinero y granos & 75 pesos \\
\hline 1892 & 10 & Maíz, dinero, machete, caballo. & 100 pesos \\
\hline
\end{tabular}




\begin{tabular}{|c|l|l|c|}
\hline $\mathbf{1 8 9 3}$ & 6 & Dinero y maíz & 20 reales \\
\hline $\mathbf{1 8 9 3}$ & 3 & Dinero & 15 pesos \\
\hline $\mathbf{1 8 9 3}$ & 3 & Cinturón de víbora, dinero, navaja. & 35 pesos \\
\hline
\end{tabular}

Fuente. Archivo Histórico del Poder Judicial del Estado de MichoacÁn, ARChivo Histórico Casa Morelos, Archivo Histórico de la Secretaría de la Defensa Nacional.

\section{Conclusiones}

En el caso del Bajío michoacano a pesar de la relativa estabilidad económica entre 1877-1893, no era la suficiente para poder satisfacer por igual ni a todos los habitantes, por ello, se buscaron alternativas para sobrellevar el contexto socioeconómico de la época por parte de los michoacanos. En este caso, el bandolerismo fue una opción oportuna y temporal para satisfacer las necesidades del tipo de sujetos que recurrieron a su ejercicio, convirtiéndose en una práctica social sumamente endémica que tuvo mayor presencia en ciertos momentos y escenarios, además de que tuvo la participaron de sujetos con un contexto socioeconómico similar, donde, los objetos y cantidades sustraídas no eran de lujo ni en amplias proporciones, porque las capacidades y protección de los bandidos se habían disminuido, en cierta medida por el fortalecimiento de la autoridad federal.

En el caso de Michoacán el bandidaje se desarrolló en niveles considerables en una región con una relativa economía estable; los bandidos ejercerían sus acciones en puntos distintos a su residencia; se robaba mayormente a quienes no eran parte de los sectores sociales altos ni parte del gobierno o sus instituciones; asimismo, los bienes robados, las cantidades y el valor de lo despojado no entendemos que constituyera una actividad con fines empresariales ni politizados. Lo anterior es porque los robos eran por temporadas bastante específicas y no iba más allá de lo que se pudiera trasladar y comercializar rápidamente (cuando no era así las gavillas tenían un número más amplio). Aunado a que al relacionar el valor de lo robado con la cantidad de sujetos que componían la gavilla las ganancias 
eran alrededor de 10-12 pesos, al no ser robos en cantidades amplias y de quita y corre, no fue un bandidaje especializado ni de larga duración.

Un aspecto relacionado con lo anterior es el hecho de aquel sujeto que recurrió al bandidaje fue plenamente por decisión personal y no de terceros, principalmente determinado por su supervivencia diaria e incluso la de su familia. Dicha práctica no necesariamente fue su primera opción, era una actividad ciertamente favorecida por el entorno y contexto socioeconómico que les rodeaba, de allí que no se denote gavillas de bandidos que salieron lejos de su espacio de cotidianidad o medio familiar. El bandidaje era una alternativa remunerable que permitía dejar lo más pronto posible la vida itinerante para regresar a su realidad como jornaleros en edad adulta, con residencia fija y familia.

Por lo tanto, el bandolerismo surgido en el Bajío michoacano no fue una elección improvisada ni llena de deseos por la aventura o lo desconocido, por el contrario, los aspectos descritos en la investigación indican una actividad llena de elecciones y maniobras, las cuales no precisamente eran un desafío, una resistencia o evasión, sino una alternativa personal y momentánea. Por lo tanto, fue un acto practicado por sujetos entendidos de lo que implicaba legalmente su ejercicio, pero sin más pretensión que la subsistencia. Por lo dicho, de acuerdo al tipo de individuos que participaron, el número de sujetos que formaban las gavillas, lo temporal de su ejercicio y el hecho de que no hubiera mayor distinción entre las víctimas, implicaba que perdió cierto contenido ideológico o de cualquier índole amplio, lo que deriva en que fuera considerado por las autoridades y la población como una acción individual de carácter adquisitivo. Al quedar limitado el margen de acción y los apoyos de terceros como las facciones políticas o los sectores específicos, tenemos que fue una acción delictiva con respaldo social mínimo. 


\section{BIBLIOGRAFIA}

APARECIDA, María (2001a), "Los patrones de criminalidad en el Estado de Chihuahua. El caso del abigeato en las últimas décadas del siglo XIX”, en Revista Historia Mexicana, México, El Colegio de México, Vol. L, Núm. 199

(2005b), "Crisis económica y desorden social en Chihuahua en vísperas de la revolución", en Romana Falcón, Culturas de pobreza y resistencia: estudios de marginados, proscritos y descontentos 1804-1910, México, El Colegio de México

ARCHER, Christon (1982), "Banditry and revolution in New Spain, 1790-1821”, en Bibliotheca Americana, United States, Vol.I, Núm.1-2

BARRETO, Carlos (2007), "Los plateados en Morelos: un ejemplo del bandolerismo en México durante el siglo XIX”, en Revista Takwá, México, Universidad de Guadalajara, Núm. 11-12

BRADING, David (1991), Mineros y comerciantes en el México borbónico (1763-1810), México, Fondo de Cultura Económica BUVE, Raymund (1971), "Movimientos campesinos mexicanos: Algunos apuntes e interrogantes sobre sus orígenes en la Sociedad Virreinal", en Anuario de Estudios Americanos, España, Escuela de Estudios Hispano-Americanos de Sevilla, Vol. XXVIII

COATSWORTH, John (1990), "Patrones de rebelión rural en América latina: México en una perspectiva comparativa”, en Friedrich Katz (Coord.), Revuelta, rebelión y Revolución. La lucha rural en México del siglo XVI al siglo XX, México, Era, Vol. I

DALE Lloyd, Jane (Coord.), (1986), Porfirio Diaz frente al descontento popular regional, 1891-1893: Antología documental, México, Universidad Iberoamericana

FALCÓN, Romana (1998), "Límites, resistencias y rompimiento del orden", en Romana Falcón y Raymond Buve (Coord.) Don Porfirio presidente...nunca omnipotente. Hallazgos, reflexiones y debates 1876-1911, México, Universidad Iberoamericana FIGUEROA Domenech, J. (1899), Guía general descriptiva de la 
República Mexicana: Historia, Geografía, Estadistica, etc. Con triple directorio del comercio y la industria, autoridades, oficinas públicas, abogados, médicos, hacendados, correos, telégrafos y ferrocarriles, etc., etc., etc. Estados y Territorios, México, Ramón Araluce, Tomo II

GANTUS, Fausta (2008), "La inconformidad subversiva: entre el pronunciamiento y el bandidaje. Un acercamiento a los movimientos rebeldes durante el tuxtepecanismo 1876-1888", en Estudios deHistoria Moderna y Contemporánea, México, Universidad Nacional Autónoma de México, Núm. 35

GARCÍA Ávila, Sergio (2010a), "La Piedad: desarrollo urbano al margen del rio Lerma, 1890-1910, en Sánchez Díaz, Gerardo (Coord.), Pueblos, villas y ciudades de Michoacán en el Porfiriato, México, Universidad Michoacana de San Nicolás de Hidalgo (2010b), "Puruándiro: Una ciudad del Bajío michoacano 18801910”, en Sánchez Díaz, Gerardo (Coord.), Pueblos, villas y ciudades de Michoacán en el Porfiriato, México, Universidad Michoacana de San Nicolás de Hidalgo

GONZÁLEZ, Luis (1989), "Ciudades y villas del Bajío colonial”, en Relaciones. Estudios de Historia y Sociedad, Zamora, El Colegio de Michoacán, Núm. 4

GUERRA, François Xavier (1991), México. Del antiguo régimen a la Revolución, México, Fondo de Cultura Económica, Vol. I

GUTIÉRREZ Puente, Beatriz (1986), “Zamora. La marginalidad urbana en una ciudad media", en Estudios Michoacanos II, México, El Colegio de Michoacán

KATZ, Friedrich (1990), "Las rebeliones rurales a partir de 1810", en Friedrich Katz (Coord.), Revuelta, rebelión y Revolución. La lucha rural en México del siglo XVI al siglo XX, México, Era, Vol. I

MAGALLANES Delgado, María del Refugio (2008), Sin oficio, beneficio ni destino: los vagos y los pobres en Zacatecas, 17861862, México, Instituto Zacatecano de Cultura

MEDINA Peña, Luis (2004), "Porfirio Díaz y la creación del sistema político en México", en Revista Istor, México, Centro de 
Investigación y Docencia Económicas, Año V, Núm. 17 MEMORIA sobre los diversos ramos de la administración pública leida ante el Congreso del Estado de Michoacán de Ocampo por el Secretario del Despacho, Morelia, Imprenta del Gobierno, 1892-1894

MEMORIA sobre los diversos ramos de la administración pública leida ante el Congreso del Estado de Michoacán de Ocampo por el Secretario del Despacho, Morelia, Imprenta del Gobierno, 1883 OCHOA, Serrano Álvaro (2010), "Jiquilpan de Juárez: bastión liberal del Occidente, 1890-1910”, en Sánchez Díaz, Gerardo (Coord.), Pueblos, villas y ciudades de Michoacán en el Porfiriato, México, Universidad Michoacana de San Nicolás de Hidalgo O’FARRILL, R. (1895), Reseña histórica estadistica y comercial de México y sus estados, México, Imprenta Regente de J. de Elizalde y Cía.

OLVEDA, Jaime (2003), Con el Jesús en la boca. Los bandidos de los altos de Jalisco, México, Universidad de Guadalajara

ORTIZ Ybarra, Héctor (1980), Puruándiro, México, Gobierno del Estado de Michoacán

PADILLA Arroyo, Antonio (2010), "Entre la ficción y la realidad. Representaciones y prácticas del bandolerismo en el Estado de Morelos a finales del siglo XIX”, en Jorge Trujillo Bretón (Coord.), En la encrucijada. Historia y marginalidad, y delito en América Latina y los Estados Unidos de Norteamérica (siglos XIX y XX), México, Universidad de Guadalajara

RANGEL, José; Ruiz, Carlos (Coord.), (2006) Discursos públicos, negociaciones y estrategias de lucha colectiva, México, El Colegio de San Luis

REINA, Leticia (1980), Las luchas populares en México, siglo XIX, México, Centro de Investigaciones y Estudios Superiores en Antropología Social (1993), "Modernización y rebelión rural en el siglo XIX", en Armando Alvarado, Guillermo (Coord.) La participación del Estado en la vida económica y social mexicana, 1767-1910, México, Instituto Nacional de Antropología e Historia 
RUIZ, Eduardo (1872), "Riqueza de Michoacán", en Boletín de la Sociedad de Geografía y Estadistica de la República Mexicana, México, Segunda Época, Tomo IV, Imprenta del Gobierno SÁNCHEZ Díaz, Gerardo (Coord.), (2010) Pueblos, villas y ciudades de Michoacán en el Porfiriato, México, Universidad Michoacana de San Nicolás de Hidalgo

SLATTA, Richard (1990), Bandits. The Varieties of Latin American Banditry, United States, Greenwood, 1987

SOLARES Robles, Laura (1999a), Bandidos somos y en el camino andamos. Bandidos. Caminos y administración de justicia en el siglo XIX. 1821-1855. El caso de Michoacán, México, Instituto Dr. José María Luis Mora

(1999b) "El bandidaje en el Estado de México durante el primer gobierno de Mariano Riva Palacio (1849-1852)", en Secuencia. Revista de Historia y Ciencias Sociales, México, Instituto Dr. José María Luis Mora, Núm. 45, 1999.

TAYLOR, William (1987), Embriaguez. Homicidio y Rebelión en las poblaciones coloniales mexicanas, México, Fondo de Cultura Económica

(1980a), "Sacarse de pobre: El bandolerismo en la Nueva Galicia 1774-1821”, en Revista Jalisco, México, Vol. 4, Núm. 3 (1990c), "Bandolerismo e insurrección: agitación rural en el centro de Jalisco. 1790-1816", en Friedrich Katz (Coord.), Revuelta, rebelión y Revolución. La lucha rural en México del siglo XVI al siglo XX, México, Era, Vol. I

TRUJILLO Bretón, Jorge Alberto (2010), "En el camino real. Representaciones. Prácticas y biografías de bandidos en Jalisco, México, 1867-1911”, en Revista Letras Históricas, México, Universidad de Guadalajara, Núm. 2

VANDERWOOD, Paul (1984), “El bandidaje en el siglo XIX. Una forma de subsistir" en Revista Historia Mexicana, México, El Colegio de México, Núm. 133

(1994) "Los bandidos de Manuel Payno", en Revista Historia Mexicana, México, El Colegio de México, Núm. 1

(1986) Desorden y progreso. Bandidos. Policias y desarrollo 
mexicano, México, Siglo XXI

VAN Young, Eric (2009), "Insurgencia y criminalidad en México 1810-1821", en Revista Memoria de las revoluciones en México, México, Instituto Dr. José María Luis Mora, Vol. 3 VERDUGO Quintero, Jorge (1991), "Heraclio Bernal: bandido social”, en Gilberto López Alanís (Coord.) El Porfiriato en Sinaloa, México, DIFOCUR

\section{Fuentes}

"Editorial. Remitidos", en La Colonia española, 6 de febrero de 1877 "El bandidaje. Necesidad de extirparlo", en El Nacional, 11 de julio de 1880 , p. 1

"Exterior", en El Diario del Hogar, 30 de noviembre de 1887.

"El bandidaje necesidad de extirparlo", en El Nacional, 11 de julio de 1880

"Remitidos", en La colonia española, 10 de febrero de 1877

"Los salteadores", en El siglo XIX, 4 mayo de 1886

"Discurso pronunciado por Manuel González el 16 de febrero de 1877 al asumir la gubernatura de Michoacán”, en El libre Sufragio, 5 de febrero de 1880

"Discurso pronunciado por Mariano Jiménez en la Ciudad de México", en El siglo XIX, 28 de septiembre 1886

"Correspondencia de Guanajuato", en La Patria, 22 de mayo de 1890

"Noticias de Puruándiro", en El Monitor republicano, 19 de agosto de 1881

"Seguridad Pública", en El siglo XIX, 14 de octubre de 1878 Archivo Histórico del Poder Judicial del Estado de Michoacán (en adelante AHPJEM), Juzgado $1^{\circ}$ Penal, Jiquilpan, "Demanda por robo en gavilla contra Brigido Mejía”, octubre de 1879

Archivo Histórico de la Secretaría de la Defensa Nacional (en adelante AHSDN), Siglo XIX, Michoacán, Exp. 481.4/12361, Foja 279, "Informe sobre defensa civil de la hacienda de Urundaneo", noviembre de 1885 
AHSDN, Siglo XIX, Michoacán, Exp. 481.4/11958, “Comunicado de jefe de la fuerza en Jiquilpan sobre actitud vecinos", Jiquilpan, 1 de abril de 1878

AHSDN, Siglo XIX, Fondo Michoacán, exp.481.4/11952, Fojas 175-176, Circular sobre combate a bandidos en Michoacán, 9 de abril de 1877

AHSDN, Siglo XIX, Fondo Michoacán, XI/481.4/12249, Foja 48, Circular para combatir bandoleros, 5 de junio 1886

Archivo Histórico Casa Morelos (en adelante AHCM), Gobierno, Policía y Guerra, Movimiento de fuerzas, Caja 264, Exp. 3, "Carta de vecinos de Zamora para que no se quite fuerza", Zamora, 23 de octubre de 1881

AHCM, Gobierno, Policía y Guerra, Movimiento de fuerzas, Caja 265, Exp. 2, "Comunicado de Ayuntamiento de Puruándiro sobre vecinos de Huango", Puruándiro, 16 de mayo de 1884

AHCM, Gobierno, Policía y Guerra, Movimiento de fuerzas, Caja 264, Exp. 1, "Oficio del ayuntamiento de Zamora sobre seguridad", Zamora, Julio de 1878

FeCHA De Recepción: 2 de agosto de 2018 Fecha de Aceptación: 6 diciembre de 2018 VERSIÓN FINAL: 7 de enero de 2019 